\title{
Clinical significance of Smac/DIABLO expression in colorectal cancer
}

\author{
KAZUYA ENDO ${ }^{1,3}$, SHUNJI KOHNOE ${ }^{2}$, AKIHIRO WATANABE ${ }^{2}$, \\ HIDEYA TASHIRO $^{1}$, HISANOBU SAKATA ${ }^{1}$, MASARU MORITA ${ }^{3}$, \\ YOSHIHIRO KAKEJI ${ }^{3}$ and YOSHIHIKO MAEHARA ${ }^{3}$
}

\author{
${ }^{1}$ Department of Surgery, Oita Prefectural Hospital, Oita; ${ }^{2}$ Department of General Surgery, \\ Fukuoka Dental College Hospital; ${ }^{3}$ Department of Surgery and Science, \\ Graduate School of Medical Sciences, Kyushu University, Fukuoka, Japan
}

Received May 15, 2008; Accepted September 9, 2008

DOI: 10.3892/or_00000229

\begin{abstract}
Second mitochondria-derived activator of caspases/ direct inhibitor of apoptosis-binding protein with low pI (Smac/DIABLO) is released by mitochondria in response to apoptotic stimuli and is thought to regulate apoptosis by antagonizing inhibitors of apoptosis proteins, which play an important role in sensitization of cancer cells to various therapeutic regimens. The expression of Smac/DIABLO has been demonstrated in various cancer cells, though little is known about its clinical significance with respect to colorectal cancer. The current study was designed to evaluate the relationship between prognosis and Smac/DIABLO expression by clinicopathological analysis of patients with colorectal cancer. Smac/DIABLO expression was evaluated using immunohistochemical staining in 121 consecutive patients with colorectal cancer and the relationship between Smac/DIABLO expression and clinicopathological factors was analyzed. Smac/DIABLO-positive expression was detected in 80 of the 121 patients $(66 \%)$. The incidence of lymph node and distant metastasis in Smac/DIABLOnegative cancer was significantly higher than that in Smac/DIABLO-positive cancer $(\mathrm{P}=0.0004$ and $\mathrm{P}=0.003$, respectively). While univariate analysis showed that survival in patients with Smac/DIABLO-negative expression was significantly poorer than in Smac/DIABLO-positive cases $(\mathrm{P}<0.0001)$, Smac/DIABLO-negative expression was a prognostic indicator independent of Dukes' staging and lymph node metastasis by multivariate analysis. This study proposes that the decrease of Smac/DIABLO expression is
\end{abstract}

Correspondence to: Dr Kazuya Endo, Department of Surgery and Science, Graduate School of Medical Sciences, Kyushu University, 3-1-1 Maidashi, Fukuoka 812-8582, Japan

E-mail:k-endo@surg2.med.kyushu-u.ac.jp

Key words: colorectal cancer, Smac/DIABLO, prognosis, immunohistochemistry an independent factor determining the poorer prognosis of patients with colorectal cancer.

\section{Introduction}

Mitochondria are vital for cellular biogenetics, playing a central role in determination of the point-of-no-return of the apoptotic process, such that they are considered to be therapeutic targets for cancer chemotherapy in recent times (1). In response to various apoptotic stimuli, mitochondria have been shown to release death proteins $(2,3)$, such as cytochrome c (cyto-c) $(4,5)$, AIF (6), Endo G (7) and HtrA2/Omi (8). Once released into the cytosol, these mitochondrial proteins trigger both caspase-dependent and -independent apoptosis. Conversely, inhibitors of apoptosis proteins (IAPs) block the activity of caspases that promote apoptotic cell death $(9,10)$ with overexpression of IAPs being related to chemoresistance in various tumor cells (11-13).

Second mitochondria-derived activator of caspases/direct inhibitor of apoptosis-binding protein with low pI (Smac/ DIABLO: SMAC hereafter) $(14,15)$ is a death protein released from mitochondria that is known to antagonize the function of IAPs (16).

Of the novel mitochondrial factors $(4-8,14,15)$, SMAC is recognized as a potent therapeutic target since it is a direct antagonist of anti-apoptotic IAPs $(17,18)$.

The level of expression of SMAC and its role in treatment sensitization has been studied in relation to several types of cancers. SMAC levels were seen to correlate well with survival in lung cancer (19), while overexpression of it increased chemosensitivity in gastric cancer cells (20), hepatocellular carcinoma (21) and osteosarcoma (22). Low levels of SMAC resulted in early resistance to chemotherapy in thyroid cancer (23) and its expression was downregulated in renal cell carcinoma, predicting a poor prognosis (24). In the case of colon cancer, increased apoptosis correlated with an increased release of SMAC in HCT116 and SW480 colon cancer cells, a couple of previous studies suggesting that decreased levels of SMAC may be important in chemoradiation-resistance in advanced colon cancer (25-27). The actual role of SMAC in colorectal cancer, however, has been ill defined. 
The current study aimed to elucidate the biological significance of SMAC expression and investigated its potential as a potent prognostic and biological marker in colorectal cancer.

\section{Patients and methods}

Patients. A total of 121 consecutive patients with colorectal cancer who had undergone surgery at Department of General Surgery, Fukuoka Dental College Hospital and its affiliated hospitals between January 1994 and May 2000 were included in this study. The study population consisted of 63 men and 58 women and their ages ranged from 31 to 84 years with a mean of 65 . Specimens were collected from the above patients, embedded in paraffin and stained to provide representative sections of each specimen. The patients were followed up and only those who died of colorectal cancer were regarded as having died of tumor-related causes. The follow-up interval after surgery ranged from 54 days to 16 years and 11 months, with a mean of 6 years and 4 months. Clinicopathological results were assessed according to the general rules for clinical and pathological studies on cancers of the colon, rectum and anus outlined by the Japanese Research Society for Cancer of the Colon and Rectum (28).

Immunohistochemical staining. Immunohistochemical staining was performed on the paraffin-embedded specimens using the peroxidase-labeled streptavidin-biotin technique with the Histone SAB-PO kit (Nichirei). Two consecutive sections of $4 \mu \mathrm{m}$ thickness were prepared from each sample. One section was stained with hematoxylin and eosin (H\&E). The other was subjected to specific immunostaining with antiSMAC antibody (IMG-248, Imgenex, San Diego, CA, USA), a polyclonal antibody that is reactive with human SMAC (29). Tissue sections were deparaffinized in xylene, rehydrated with a series of graded ethanols and placed in phosphatebuffered saline (PBS) for $10 \mathrm{~min}$. After cooking the slides in a pressure cooker in citrate buffer solution ( $\mathrm{pH} \mathrm{6.0)}$ ) for $6 \mathrm{~min}$, to retrieve the antigen, endogenous peroxidase activity was blocked for 30 min with methanol containing $0.3 \%$ hydrogen peroxidase. Sections were then incubated with $10 \%$ nonimmunized rabbit serum for $10 \mathrm{~min}$ to block nonspecific binding of the immunoreagents and incubated overnight at $4{ }^{\circ} \mathrm{C}$ with rabbit anti-human polyclonal SMAC antibody at a 1:50 dilution. The sections were subsequently incubated with a second stage biotinylated antibody for $20 \mathrm{~min}$, followed by incubation with horseradish peroxidase-labeled streptavidin for $20 \mathrm{~min}$ at room temperature. After washing in PBS, localization of SMAC was visualized with diaminobenzidine tetrahydrochloride.

All the stained sections were analyzed by two observers. In addition, the results were assessed by a pathologist, who had not been given any clinical information about the sections. If the percentage of positive-staining cancer cells accounted for $<30 \%$ of the total number of cancer cells, the respective patients from whom the specimens had been collected were classified as SMAC-negative, while if the number of stained cells exceeded $30 \%$, patients were classified as SMAC-positive regardless of the intensity of staining. $\mathbf{a}$

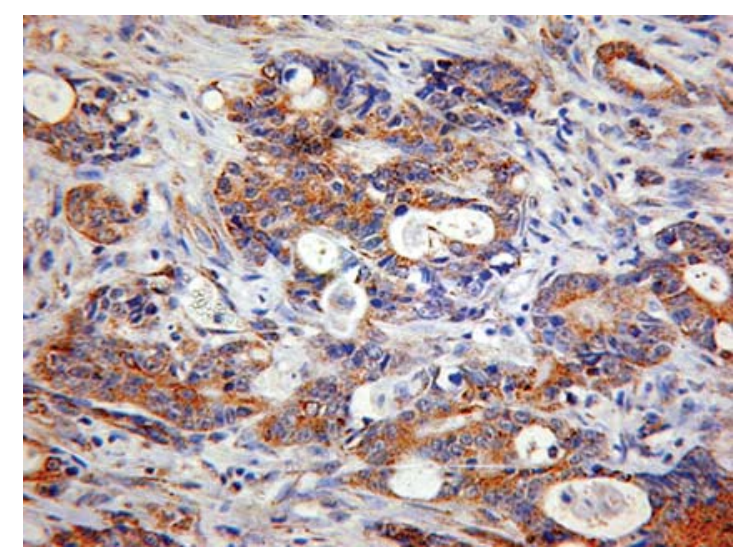

b

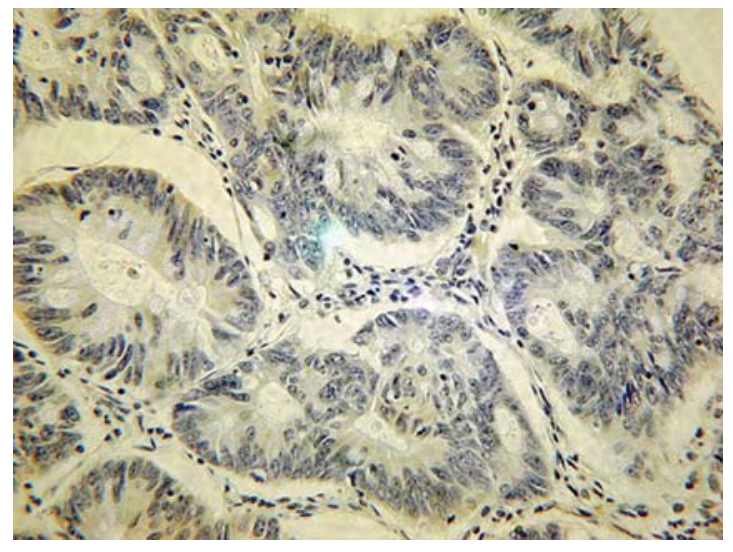

Figure 1. (a) SMAC-positive immunostaining of moderately differentiated adenocarcinoma of the colon showing cytoplasmic expression of carcinoma cells (x200). (b) SMAC-negative immunostaining in moderately differentiated adenocarcinoma of the colon (x200).

Statistical analysis. The 121 patients were divided into two groups, depending on whether Smac/DIABLO expression was positive or negative and compared for clinicopathological characteristics using the Chi-square and Student's t-tests. Cumulative survival rates were evaluated by the Kaplan-Meier method and the survival curves were tested by the Mantel-Cox method. Multivariate survival analysis was done according to Cox's proportional hazards model in a forward stepwise manner. A p-value $<0.05$ was considered statistically significant.

\section{Results}

SMAC was predominantly localized in the cytoplasm of cancer cells (Fig. 1a and b). The relationship between SMAC expression and the clinicopathological features of the patients are shown in Table I. Based on the results of immunohistochemical staining, 80 of 121 patients $(66 \%)$ were positive for SMAC expression.

Clinicopathological features between SMAC-positive and -negative groups were compared. The incidence of lymph node metastasis was $15 / 80(18.75 \%)$ in the SMAC-positive group, which was significantly lower than the 19/41 (46.3\%) in the SMAC-negative group $(\mathrm{P}=0.003)$. Distant metastases, 
Table I. SMAC expression and clinicopathological characteristics.

\begin{tabular}{|c|c|c|c|}
\hline Variables & SMAC-positive $(\mathrm{n}=80)(\%)$ & SMAC-negative $(\mathrm{n}=41)(\%)$ & P-value \\
\hline \multicolumn{4}{|l|}{ Gender } \\
\hline Male & $45(56.25)$ & $18(43.9)$ & \multirow[t]{2}{*}{ N.S. } \\
\hline Female & $35(43.75)$ & $23(56.1)$ & \\
\hline Age (years) & $64.5 \pm 12.2$ & $67.3 \pm 11.8$ & N.S. \\
\hline Tumor size (mm) & $49.1 \pm 20.4$ & $51.2 \pm 25.3$ & N.S. \\
\hline \multicolumn{4}{|l|}{ Location of tumors } \\
\hline Cecum and ascending colon & 14 (17.5) & $7(17.1)$ & \multirow[t]{5}{*}{ N.S. } \\
\hline Transverse colon & $8 \quad(10.0)$ & $3(7.3)$ & \\
\hline Descending colon & $4 \quad(5.0)$ & $1 \quad(2.4)$ & \\
\hline Sigmoid colon & $26 \quad(32.5)$ & $19(46.4)$ & \\
\hline Rectum & $28 \quad(35.0)$ & $11(26.8)$ & \\
\hline \multicolumn{4}{|l|}{ Pathological type } \\
\hline Well & $45(56.25)$ & $24(58.5)$ & \multirow[t]{4}{*}{ N.S. } \\
\hline Moderately & $23(28.75)$ & $14(34.2)$ & \\
\hline Poorly & $5(6.25)$ & $2(4.9)$ & \\
\hline Mucinous & $7 \quad(8.75)$ & $1 \quad(2.4)$ & \\
\hline \multicolumn{4}{|l|}{ Tumor depth } \\
\hline Mucosa & $3 \quad(3.75)$ & $1 \quad(2.4)$ & \multirow[t]{6}{*}{ N.S. } \\
\hline Submucosa & $6 \quad(7.5)$ & $2(4.9)$ & \\
\hline Muscularis & $13(16.25)$ & $4 \quad(9.8)$ & \\
\hline Subserosa & $25(31.25)$ & $8(19.5)$ & \\
\hline Serosa & $31(38.75)$ & $20(48.8)$ & \\
\hline Invading surrounding organs & $2 \quad(2.5)$ & $6(14.6)$ & \\
\hline \multicolumn{4}{|l|}{ Lymph node metastasis } \\
\hline Positive & $15(18.75)$ & $19(46.3)$ & \multirow[t]{2}{*}{0.003} \\
\hline Negative & $65(81.25)$ & $22(53.7)$ & \\
\hline \multicolumn{4}{|l|}{ Lymphatic permeation } \\
\hline Positive & $18 \quad(22.5)$ & $10(24.4)$ & \multirow[t]{2}{*}{ N.S. } \\
\hline Negative & $62 \quad(77.5)$ & $31(75.6)$ & \\
\hline \multicolumn{4}{|l|}{ Venous invasion } \\
\hline Positive & $20 \quad(25.0)$ & $13(31.7)$ & \multirow[t]{2}{*}{ N.S. } \\
\hline Negative & $60 \quad(75.0)$ & $28(68.3)$ & \\
\hline \multicolumn{4}{|l|}{ Distant metastasis } \\
\hline Positive & $1 \quad(1.25)$ & $9(22.0)$ & \multirow[t]{2}{*}{0.0004} \\
\hline Negative & $79(98.75)$ & $32(78.0)$ & \\
\hline \multicolumn{4}{|l|}{ Dukes' classification } \\
\hline A & $19(23.75)$ & $6(14.6)$ & \multirow[t]{4}{*}{0.0001} \\
\hline B & $45(56.25)$ & $13(31.7)$ & \\
\hline $\mathrm{C}$ & $15(18.75)$ & $13(31.7)$ & \\
\hline $\mathrm{D}$ & $1 \quad(1.25)$ & $9(22.0)$ & \\
\hline
\end{tabular}

N.S., not significant; well, well differentiated adenocarcinoma; modereately, moderately differentiated adenocarcinoma; poorly, poorly differentiated adenocarcinoma; mucinous, mucinous adenocarcinoma and SMAC, Smac/DIABLO.

including liver and lung metastasis, were recognized in a total of 10 patients, 9 of whom belonged to the SMAC-negative group. Tumor staging according to Dukes' criteria was performed. The proportion of patients classified as stage $\mathrm{C}$ and D in the SMAC-positive group was significantly lower than that in the negative group $(16 / 80,20.0 \%$ and $22 / 41$,
$53.7 \%$, respectively) while the proportion of patients classified as stage A and B was significantly higher in the positive as compared to the negative group $(64 / 80,80.0 \%$ and 19/41, 46.3\%; $\mathrm{P}=0.0001)$. No significant differences were noted between the two groups with respect to tumor size, tumor location, histological differentiation and lymphatic and 
Table II. Factors independently associated with prognosis.

\begin{tabular}{lcccc}
\hline Variables & Standard error & Odds ratio & CI 95\% & P-value \\
\hline Lymphatic invasion & 0.510 & 0.577 & $0.274-2.025$ & 0.564 \\
Venous invasion & 0.509 & 0.699 & $0.258-1.899$ & 0.484 \\
SMAC expression & 0.569 & 4.460 & $4.150-38.659$ & $<0.001$ \\
Dukes' stage & 0.829 & 3.320 & $0.013-0.324$ & $<0.001$ \\
Lymph node metastasis & 0.758 & 2.516 & $1.524-29.777$ & 0.012 \\
\hline
\end{tabular}

CI, confidence interval and SMAC, Smac/DIABLO.

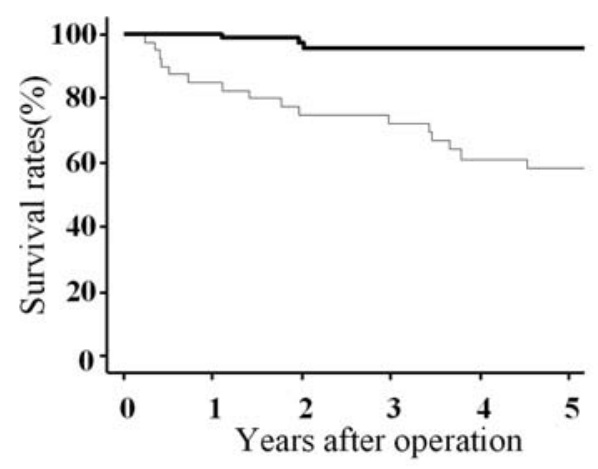

Figure 2. Survival curve of colorectal cancer in SMAC exhibiting and non-exhibiting patients. The prognosis of patients was significantly worse in SMAC-negative patients (thin line) as compared to patients who were SMAC-positive (thick line $)(\mathrm{P}<0.0001)$.

venous invasion. Thus, negative expression of SMAC correlated with aggressiveness of the cancer.

One-, 3- and 5-year survival rates of patients in the SMAC-positive group were 98.7,96.0 and 96.0\%, respectively, which were significantly higher than survival rates of patients in the SMAC-negative group $(82.5,72.2$ and $58.1 \%$, respectively; $\mathrm{p}<0.0001$ ) (Fig. 2). Multivariate analysis demonstrated that SMAC expression, Dukes' staging and lymph node metastasis were independent prognostic factors in patients with colorectal cancer (Table II).

\section{Discussion}

The data presented here indicate that SMAC expression significantly correlated with lymph node metastasis, distant metastasis and Dukes' staging. Furthermore, it was an independent prognostic factor taking rank with lymph node metastasis and Dukes' staging, the expression of SMAC being inversely related to the aggressiveness of the cancer. A strong explanation for the above results is the close relationship between SMAC expression and its ability to regulate apoptosis.

IAPs such as XIAP are strongly expressed in various cancers including colon cancer (30) and are associated with poor prognosis and resistance to apoptosis $(11,31)$. XIAP is the most potent and best characterized member of the mammalian IAP family (32), being a predominant SMAC binding protein. SMAC binds to XIAP, displaces XIAP from caspase-9, promotes cleavage of effector caspases and induces apoptosis $(33,34)$. Several previous studies have shown that increased apoptosis correlates with an increased release of SMAC in colon cancer cells (25-27).

Hence, it can be safely hypothesized that decreased or lack of SMAC expression is related to increased aggressiveness of a cancer and that SMAC modulates malignant behavior through interaction with IAPs.

Another explanation in support of SMAC's important role in cancer may be its ability to regulate the metastatic potential of a cancer. Huerta et al reported that SMAC expression was diminished in metastatic colon cancer cells, making it a potential target for chemosensitization in the treatment of advanced colon cancer (35).

In the current study, 9 out of 10 cases with distant metastasis had negative expression of SMAC. Distant or lymph node metastases are significant prognostic factors in colon cancer (36), reflecting the ability of SMAC to affect the progression of cancer through promoting metastasis.

Many studies relating to SMAC and colon cancer have suggested that stimulation of apoptosis causes concomitant release of three important mitochondrial pro-apoptotic factors with different mechanisms of action: a) release of cytochrome c, which mediates caspase- 3 activation with formation of the apoptosome; b) release of AIF which mediates DNA fragmentation through caspase-independent pathway; and c) release of SMAC, which neutralizes the activity of IAPs. The relative role and ratios of these factors in the process of apoptosis are important, though as yet unclear (4-6,37). SMAC is therefore believed to regulate carcinogenesis in colon cancer by virtue of its ability to neutralize IAPs.

In conclusion, immunohistochemical detection of SMAC expression is a potent prognostic marker in colorectal cancer, although its biological function is still not completely clear. Elucidation of such a function may contribute to our search for a new strategy against cancer progression and metastasis. Recent studies with SMAC peptides (SMAC-mimetics) have shown that it could be useful in the development of more effective chemopreventive strategies and agents for various types of cancer cells including colon cancer $(17,18,38-40)$. This may lead to human trials with specific therapies targeting SMAC in advanced or inoperable colorectal cancers refractory to conventional chemoradiotherapies.

\section{Acknowledgements}

We are grateful to Dr Shogo Urabe of the Department of Pathology, Oita prefectural hospital, Japan for discussions related to pathology. 


\section{References}

1. Galluzzi L, Larochette N, Zamzani N and Kroemer G: Mitochondria as therapeutic target for cancer chemotherapy. Oncogene 25: 4812-4830, 2006.

2. Bossy-Wetzel E and Green DR: Apoptosis: checkpoint at the mitomchondrial frontier. Mutat Res 434: 243-251, 1999.

3. Kromer G and Reed JC: Mitochondrial control of cell death. Nat Med 6: 513-519, 2000.

4. Kluck RM and Kashibatta S: A lively meeting of a deathly topic. Apoptosis 2: 337-342, 1997.

5. Yang J, Liu X, Balla K, Gillenwiter A, et al: Prevention of apoptosis by Bcl-2-Release of cytochrome-c from the mitochondria blocked. Science 275: 1129-1136, 1997.

6. Susin SA, Zamazami N, Castedo M, et al: Bcl-2 inhibits the mitochondrial release of an apoptogenic protease J Exp Med 184: 1331-1341, 1996.

7. Parrish L, Li K, Klotz D, et al: Mitochondrial endonuclease G is important for apoptosis in C. elegans. Nature 412: 90-94, 2001.

8. Hedge R, Srinivasula SM, Zhang Z, et al: Idetification of $\mathrm{Omi} / \mathrm{HtrA} 2$ as a mitochondrial apoptotic serine protease that disrupts inhibitor of apoptosis protein-caspase interaction. J Biol Chem 277: 432-438, 2002.

9. Thornberry NA and Lazebnik Y: Caspases: enemies within. Science 281: 1312-1316, 1998.

10. Harada H and Grant S: Apoptosis regulators. Rev Clin Exp Hematol 7: 117-138, 2003.

11. Deveraux QL and Reed JC: IAP family proteins - suppressors of apoptosis. Genes Dev 13: 239-252, 1999.

12. Tamm I, Kornblau SM, Segall H, et al: Expression and prognostic significance of IAP-family genes in human cancers and myeloid leukemias. Clin Cancer Res 6: 1796-1803, 2000.

13. Krajewska M, Krajewski S, Banares S, et al: Elevated expression of inhibitor of apoptosis proteins in prostate cancer. Clin Cancer Res 9: 4914-4925, 2003.

14. Du C, Fang M, Li Y, Li L and Wang X: Smac, a mitochondrial protein that promotes cytochrome c-dependent caspase activation by eliminating IAP inhibition. Cell 102: 33-42, 2000.

15. Verhagen AM, Ekert PG, Pakusch J, et al: Idetification of DIABLO, a mammalian protein that promotes apoptosis by binding to and antagonizing IAP proteins. Cell 102: 43-53, 2000.

16. Wu G, Chai J, Suber TL, et al: Structual basis of IAP recognition by Smac/DIABLO. Nature 408: 1008-1012, 2000.

17. Van Ginkel PR, Sareen D, Subramanian L, et al: Resveratrol inhibits tumor growth of human neuroblastoma and mediates apoptosis by directly targeting mitochondria. Clin Cancer Res 13: 5162-5169, 2007.

18. Weisberg E, Kung AL, Wright RD, et al: Potentiation of antileukemic therapies by Smac mimetics, LBW242: effects on mutant FLT3-expreaaing cells. Mol Cancer Ther 6: 1951-1961, 2007.

19. Sekimura A, Konishi A, Mizuno K, et al: Expression of Smac/ DIABLO is a novel prognostic marker in lung cancer. Oncol Rep 11: 797-802, 2004

20. Zheng LD, Tong QS, Wang L, Liu J and Qian W: Stable transfection of extrinsic Smac gene enhances apoptosis of chemotherapeutic drugs on gastric cancer cells. World J Gastroenterol 11: 79-83, 2005.

21. Bao ST, Gui SQ and Lin MS: Relationship between expression of Smac and Survivin and apoptosis of primary hepatocellular carcinoma. Hepatobiliary Pancreat Dis Int 5: 580-583, 2006.

22. Zhao J, Jin J, Zhang X, et al: Transfection of Smac sensitizes tumor cells to etoposide-induced apoptosis and eradicates ectablished human hepatoma in vivo. Cancer Gene Ther 13: 420-427, 2006
23. Tirro E, Consoli ML, Massimino M, et al: Altered expression of c-IAP1, survivin and Smac contributes to chemotherapy resistance in thyroid cancer cells. Cancer Res 66: 4263-4272, 2006.

24. Mizutani Y, Nakanishi H, Yamamoto K, et al: Downregulation of Smac/DIABLO expression in renal cell carcinoma and its prognostic significance. J Clin Oncol 23: 448-454, 2005.

25. Rashmi R, Kumar S and Karunagaran D: Human colon cancer cells lacking Bax resist curcumin-induced apoptosis and Bax requirement is dispensable with ectopic expression of Smac or downregulation of Bcl-XL. Carcinogenesis 26: 713-723, 2005.

26. Sinicrope FA, Penington RC and Tang XM: Tumor necrosis factor-related apoptosis - inducing ligand-induced apoptosis is inhibited by Bcl-2 but restored by small molecule Bcl-2 inhibitor, HA 14-1, in human colon cancer cells. Clin Cancer Res 10: 8284-8292, 2004

27. Kohli M, Yu J, Seaman C, et al: Smac/DIABLO-dependent apoptosis induced by nonsteroidal anti-inflammatory drugs(NSAIDs) in colon cancer cells. Proc Natl Acad Sci USA 101: 16897-16902, 2004.

28. Japanese Research Society of Cancer of the Colon and Rectum: General rules for clinical and pathologic studies on cancer of the colon, rectum and anus. Jpn J Surg 13: 557-573, 1983.

29. Ren Y, Akyurek N, Schlette E, Rassidakis GZ and Medetros LJ: Expression of Smac/DIABLO in B-cell non-Hodkin and Hodgkin lymphomas Hum Pathol 37: 1407-1413, 2006.

30. Cummins JM, Kohli M, Rago C, Kinzler KW, Vogelstein B and Bunz F: X-Linked Inhibitor of Apoptosis Protein(XIAP) is a nonredundant modulator of tumor necrosis factor-related apoptosis-inducing ligand(TRAIL)-mediated apoptosis in human cancer cells. Cancer Res 64: 3006-3008, 2004.

31. Sasaki H, Sheng Y, Kotsuji F, et al: Down regulation of X-linked inhibitor of apoptosis protein induces apoptosis in chemoresistant human ovarian cancer cells. Cancer Res 60: 5659-5666, 2000.

32. Zhang HG, Wang J, Yang X, Hsu HC and Mountz JD: Regulation of apoptosis proteins in cancer cells by ubiquitin. Oncogene 23: 2009-2015, 2004.

33. Goyal L: Cell death inhibition: Keeping caspases in check. Cell 104: 805-808, 2001.

34. Srinivasula SM, Hegde R, Saleh A, et al: A conserved XIAPinteraction motif in caspase-9 and Smac/DIABLO regulates caspase activity and apoptosis. Nature 410: 112-116, 2001.

35. Huerta S, Heinzerling JH, Anguiano-Hernandez YM, et al: Modification of gene products involved in resistance to apoptosis in metastatic colon cancer cells: Roles of Fas, Apaf-1, NFkB IAPs, Smac/DIABLO, and AIF. J Surg Res 142: 184-194, 2007.

36. Ogata $\mathrm{Y}$, Torigoe $\mathrm{S}$, Matono $\mathrm{K}$, et al: Prognostic factors after potentially curative resection in stageII or III colon cancer. Kurume Med J 52: 67-71, 2005.

37. Anguiano-Hernandez YM, Chartier A and Huerta S: Smac/ DIABLO and colon cancer. Anticancer Agents Med Chem 7: 467-473, 2007.

38. Gao Z, Tian Y, Wang J, et al: A dimeric Smac/Diablo peptide directly relieves caspase-3 inhibition by XIAP. Dynamic and cooperative regulation of XIAP by Smac/Diablo. J Biol Chem 282: 30718-30727, 2007.

39. Gaither A, Porter D, Yao Y, et al: A Smac mimetic rescue screen reveals roles for inhibitor of apoptsis protein in tumor necrosis factor-a signaling. Cancer Res 67: 11493-11498, 2007.

40. Bank A, Wang P, Du C, Yu J and Zhang L: Smac mimetics sensitize nonsteroidal anti-inflammatory drug induced apoptosis by promoting caspase- 3 mediated cytochrome c release. Cancer Res 68: 276-284, 2008. 